\title{
A TREATISE ON DIFFERENTIAL EQUATIONS
}

BY

GEORGE BOOLE, F. R. S.

Cambridge 1859 , vol. in 8. pag. I-XY. 1- $\$ 85$.

Questa nuova, el interessante opera testè pubblicata dal Sig. G. Boole c̀ divisa in dieciotto capitoli, dei quali la maggior parte viene impiegata per l' integrazione delle così dette Equazioni aifferenziali, ed il rimanente per l'Equazioni a derivate parziali. Alla fine di ogni capitolo poi si propongono degli esempi a sviluppo delle tcorie. Quanto possa essere rilevante quest'opera nello stato attuale della scienza to indica il titolo, ed il nome dell'illustre geometra d'Irlanda. Non essendo mio scopo in questa breve notizia, e quasi puramente bibliografica di venire all'esame, ed alla discussione dei metodi usati dal Sig. Boole mi basterà notare, chc esso mettc gindiziosamente a profitto, quanto di più importante su quesl'argomento è stato scritto da Eulero, Lagrange, Laplace, Legendre, Cauchy, Jacobi, Hamilton, e dall'autore medesimo nelle transazioni filosofiche di Londra. In questa circostanza mi piacerà anche rammentare le dotte Memorie degli illustri geometri italiani Paoli, Brunacci, Malfatti, pubblicate già da molti anni a questa parte. Nei capitoli XVI e XVII vienc sviluppato dal Sig. Boole l'uso dei simboli per l'integrazione dell'equazioni. L'autore espone con un bel ordine e chiarezza le varie formole simboliche, e richiama in special modo un suo lavoro fin dal 1844, e differenti pubblicazioni di altri distinti geometri inglesi. Certamente l'uso dei simboli, e delle caratteristiche messo in opera con quelle cautele, e precauzioni che si richiedono è di una mirabile risorsa nella risoluzione di un gran numero di problemi di analisi, e di Calcolo Integrale. Io citerò le belle ed eleganti Memorie del Sig. Cauchy per l'integrazione dell'Equazioni lineari, argomento che anche io per mezzo del Calcolo dei Residui, e coll'uso dei simboli, e delle caratteristiche diffusamente tratlai fin dal 1842 , e 1843 in una serie di Memorie pubblicate nel Giornale Arcadico di Roma, e più recentemente nel 1854 in una mia Memoria nel tom. 25 della Società Italiana. Infine nell'ultimo capitolo il Sig. Boole succintamente espone la soluzione dell'equazioni lineari per mezzo degli Integrali definili: i metodi sono quei di Laplace, Fourièr, Poisson, Cauchy, ed io pure con qualche estensione mi proposi lo stesso soggetto nelle citate Memorie del 1842, 1843. Utilissima riuscirà la lettura dell'opera del Sig. Boole tanto ai Professori quanto agli allievi, e per una maggior diffusione sarebbe da augurarsi una buona traduzione in una lingua più diffusamente conosciuta. Terminerò questa breve notizia con dire, che essa potrà anche servir di guida ad altri geometri, che sopra l'equazioni differenziali si proponessero il più grande sviluppo.

B. $T$. 